\title{
Paralelismo multi-nível: uma revisão sistemática da literatura*
}

\author{
Natiele Lucca $^{1}$, Claudio Schepke ${ }^{1}$ \\ ${ }^{1}$ Programa de Pós-Graduação em Engenharia de Software \\ Laboratório de Estudos Avançados em Computação (LEA) \\ Universidade Federal do Pampa (UNIPAMPA) - Campus Alegrete \\ 97546-550 - Alegrete - RS - Brazil \\ natielelucca@gmail.com, claudioschepke@unipampa.edu.br
}

\begin{abstract}
Resumo. O paralelismo multi-nível explora diferentes níveis de abstração de hardware a fim de melhorar o desempenho de aplicações. Ferramentas de programação são destinadas normalmente a apenas um nível de paralelismo, destacando-se a ausência de ferramentas que exploram outros níveis de abstração. Este trabalho apresenta uma revisão sistemática da literatura que investiga ferramentas e frameworks que exploram o paralelismo multinível.
\end{abstract}

\section{Introdução}

De acordo com [Schepke and Maillard 2012] o paralelismo pode ser expresso internamente em um processador, através das arquiteturas multicores; interno a um computador, usando multiprocessadores, Graphics Processing Units (GPUs) e Field-Programmable Gate Arrays (FPGAs); e entre computadores. Cada aplicação tem suas características particulares que se adaptam de diferentes formas quando aplicadas a diferentes níveis de paralelismo. O desafio é estabelecer o nível de paralelismo ideal para a aplicação. O objetivo deste trabalho é realizar uma revisão sistemática da literatura para identificar frameworks ou ferramentas que utilizam paralelismo multi-nível em aplicações.

\section{Planejamento da revisão sistemática da literatura}

A revisão foi realizada nas bases de dados: $\mathrm{ACM}^{1}$, $\mathrm{IEEE}^{2}$, Science Direct $^{3}$, Scopus $^{4}$ e Springer Link ${ }^{5}$. As questões de pesquisa são a) Identificar frameworks ou ferramentas com paralelismo multi-nível; b) Quais são as características e limitações dos frameworks ou ferramentas identificados?; e c) Os frameworks ou ferramentas apresentam quais níveis de paralelismo?. A estratégia se seleção de estudos foi ler o título as palavras-chaves e o abstract para aplicar os critérios de inclusão e exclusão.

A string genérica aplicada foi: ("multi-level parallelism"OR "multilevel parallelism") AND ("performance"OR "performance gain"OR "high-performance"OR "high performance") AND (framework OR application OR tool).

*O presente trabalho foi desenvolvido no Laboratório de Estudos Avançados (LEA) com apoio AGP 2020 - Pós-Graduação/Unipampa 2020.

${ }^{1}$ https://dl.acm.org/

${ }^{2} \mathrm{https}: / /$ ieeexplore.ieee.org/Xplore/home.jsp

${ }^{3} \mathrm{https} / / / \mathrm{www}$. sciencedirect.com/

${ }^{4}$ https://www.scopus.com/home.uri

${ }^{5}$ https://link.springer.com/ 
Os critérios de inclusão de artigos definem as características que os artigos devem conter. Para tanto foram aplicados: a) O estudo apresenta um novo framework/ ferramenta/aplicação de paralelismo multi-nível. b) O estudo apresenta um framework/ ferramenta/aplicação existente de paralelismo multi-nível. c) O estudo apresenta um comparativo entre frameworks/ferramentas/aplicações de paralelismo multi-nível.

Os critérios de exclusão definem as características de rejeição de artigos. Os critérios de exclusão aplicados no estudo são: a) Estudo duplicado. b) Estudo em um idioma distinto do inglês. c) Estudo que não apresenta framework/ferramenta/aplicação de paralelismo multi-nível. d) Estudo indisponível na base de dados.

Os critérios de qualidade aplicados no trabalho são: a) $\mathrm{O}$ estudo descreve o framework/ferramenta/aplicação? b) $\mathrm{O}$ estudo apresenta trabalhos relacionados a outros frameworks/ferramentas/aplicações de paralelismo multi-nível? c) O estudo relata os níveis de abstração utilizados no framework/ferramenta/aplicação? d) O estudo compara os testes realizados no framework/aplicação/ferramenta com o realizado em outros?

\section{Resultados e Considerações Finais}

As bases ACM, IEEE, SCIENCE DIRECT, SCOPUS e SPRINGER LINK retornaram, respectivamente 110, 42, 109, 167 e 222 artigos. No total foram 650 artigos selecionados, destes 17 foram aceitos, 577 foram rejeitados, 56 foram removidos (51 eram duplicados e 5 eram inconsistentes).

Foram submetidos à avaliação de qualidade 17 artigos. Destes, 10 foram aceitos e 5 foram rejeitados (por não respeitar os critérios estabelecidos). Os critérios de qualidade atribuem valor a um artigo, descrevendo os artigos como ótimos quando atendem aos requisitos, intermediários que atendem parcialmente aos requisitos e ruim, quando não atendem aos requisitos da pesquisa.

Após aplicar a extração de dados, as ferramentas e bibliotecas identificadas na pesquisa são: GridPACK, PPF (A Parallel Particle Filtering Library), XKaapi, Anthill, CAPO, Cilk, StarSs, OmpSs, Athapascan, KAAPI, StarPU, SMPSs, PLASMA, FLAME, MAGMA, DAGuE, QUARK, REWORK e GPUSs.

As questões de pesquisa que buscam as características e limitações dos frameworks ou das ferramentas ou de aplicações envolvem mais pesquisa, pois os artigos não descrevem as ferramentas. Os estudos justificam o uso para ganho de desempenho e redução do tempo de execução dos algoritmos envolvidos.

Por fim, na questão de pesquisa sobre paralelismo apenas alguns estudos descrevem o paralelismo que contém o framework. Dos artigos que descrevem são aplicados as APIs OpenMP, MPI, OpenCL, CUDA, SIMD e Java threads.

\section{Referências}

Schepke, C. and Maillard, N. (2012). Exploring multi-level parallelism in atmospheric applications. In 2012 13th Symposium on Computer Systems, pages 41-48. IEEE. 